\title{
Microwave Background Constraints on Mixing of Photons with Hidden Photons
}

\author{
Alessandro Mirizzi \\ Max-Planck-Institut für Physik (Werner Heisenberg Institut) \\ Föhringer Ring 6, 80805 München, Germany \\ Javier Redondo \\ Deutsches Elektronen Synchrotron \\ Notkestraße 85, 22607 Hamburg, Germany
}

Günter Sigl

II. Institut für theoretische Physik, Universität Hamburg, Luruper Chaussee 149, 22761 Hamburg, Germany

\begin{abstract}
Various extensions of the Standard Model predict the existence of hidden photons kinetically mixing with the ordinary photon. This mixing leads to oscillations between photons and hidden photons, analogous to the observed oscillations between different neutrino flavors. In this context, we derive new bounds on the photon-hidden photon mixing parameters using the high precision cosmic microwave background spectral data collected by the Far Infrared Absolute Spectrophotometer instrument on board of the Cosmic Background Explorer. Requiring the distortions of the CMB induced by the photon-hidden photon mixing to be smaller than experimental upper limits, this leads to a bound on the mixing angle $\chi_{0} \lesssim 10^{-7}-10^{-5}$ for hidden photon masses between $10^{-14} \mathrm{eV}$ and $10^{-7} \mathrm{eV}$. This low-mass and low-mixing region of the hidden photon parameter space was previously unconstrained.
\end{abstract}

Keywords: hidden photons, cosmic microwave background.

PACS numbers: 98.80.Cq, 98.80.Es, 98.70.Vc, 14.70.Pw 


\section{Introduction}

The last decade has witnessed the blooming of modern cosmology, supported by a consolidated model of particle physics and a huge amount of new observational data with unprecedented precision (see for example the latest WMAP team results $[1,2]$ ). Every observation has been shown to fit with the predictions of the so-called $\Lambda$-CDM model, a spatially flat Friedman universe, with $\sim 10^{-8}$ baryons per photon and whose energy composition is now dominated by two unknown ingredients: dark matter and dark energy. At this stage of concordance, observational cosmology can be used to test the existence of non-standard particle physics.

One of the most powerful probes used to constrain exotic physics is represented by the observations of the cosmic microwave background radiation. Standard cosmology predicts relic radiation from the big bang which, being originally in thermal equilibrium with matter, suffered a soft decoupling and, therefore, still features today a perfect black body spectrum. As it turns out, during most of the history of the universe this perfect blackbody has been completely unprotected against distortions. Therefore, any new particle beyond the standard model which can interact with photons can potentially distort the blackbody spectrum, providing us with a glimpse of the existence of such a particle.

These distortions are expected to be more severe if the new particles have feeble couplings, so that their interactions with photons do not allow to establish thermal equilibrium (but of course strong enough to produce some effect), and if they have small masses, such that photons can annihilate into them and/or scattering processes are not suppressed by a heavy mass scale. Since the cosmic radiation spectrum freezes out when the temperature of the universe falls below the $\sim \mathrm{eV}$ scale, the spectrum of the cosmic microwave background (CMB) can, therefore, be an excellent probe for weakly interacting sub-eV particles (WISPs).

Among the most elusive WISPs we find low mass hidden photons (HPs), gauge bosons of a $\mathcal{U}(1)_{\text {hid }}$ gauge symmetry having kinetic mixing with the ordinary photon. Due to the mixing one expects photon-HP oscillations driven by the mass difference of the two particles. Clearly, as this mass becomes smaller the oscillation length grows and oscillations are harder to detect. In this respect, the CMB provides an excellent probe for photon oscillations into low mass HPs, since the beam-line is the longest at our disposal, namely the whole universe. Indeed, hidden photons were the first WISP candidates to be confronted with CMB data in the early 80's [3].

Recently, new intriguing ideas and experimental techniques have been proposed to achieve possible detections of these still elusive particles [4-6]. Moreover, the astrophysical and cosmological role of HPs is currently under exploration [7-10].

The purpose of this paper is to revisit the CMB bounds on photon-HP mixing using the most precise available observations of the CMB spectrum, those provided by the Far Infrared Absolute Spectrophotometer (FIRAS) on board of the Cosmic Background Explorer (COBE) [11,12]. The high precision of this measurement, which confirmed 
the blackbody nature of the spectrum at better than 1 part in $10^{4}$, has been already exploited to constrain hidden photons [13] and also other WISPs such as axions [14], or radiative neutrino decays [15] or millicharged particles [16]. However, the already existing constraints $[3,13]$ did not properly take into account the refractive properties of the primordial plasma and are thus incomplete. The medium effects are especially important when we realize that a resonant photon-HP conversion is possible, similar to the Mikheev-Smirnov-Wolfenstein (MSW) effect in the neutrino case [17-19]. This resonant conversion is much stronger than the vacuum oscillations considered so far and, therefore, the bounds we obtain are much stronger.

The plan of our work is as follows. In Section 2 we summarize the relevant formalism concerning the photon-HP mixing. In particular, we describe how the medium effects modify the oscillations in our system. In Section 3 we present our analytical recipe to calculate the photon-HP conversion probability across a resonance in the expanding universe. In Section 4 we describe our simplified model for the effective photon mass, induced by the primordial plasma. In Section 5 we describe the constraints coming from spectral CMB distortions. In Section 6 we outline in a general way the strength of photon-HP mixing bounds that can be obtained from an observed modification of the photon flux from a generic astrophysical source. Finally, in Section 7 we compare our new cosmological bound with the other ones existing in the literature and we draw our conclusions.

\section{Photon-Hidden Photon Mixing}

A hidden photon is the gauge boson of a gauge $\mathcal{U}(1)_{\text {hid }}$ symmetry under which all the SM fields are uncharged, and thus remains hidden in our world. At energies above the electroweak scale, particles charged under $\mathcal{U}(1)_{\text {hid }}$ and the SM hypercharge are likely to exist and act as messengers between the two "sectors" of low-energy physics. Their effects will produce effective operators in the low-energy theory that contain the SM and the hidden photon. Typically these operators are irrelevant, suppressed by the heavy particle masses. But there is still one marginal operator, the so called kinetic mixing [20] whose effects are not in principle suppressed by heavy masses and thus can lead to relatively large effects. The low energy Lagrangian of such an extension of the SM would be then [8]

$$
\begin{aligned}
\mathcal{L} & =-\frac{1}{4} F_{\mu \nu} F^{\mu \nu}-\frac{1}{4} B_{\mu \nu} B^{\mu \nu} \\
& +\frac{\sin \chi_{0}}{2} B_{\mu \nu} F^{\mu \nu}+\frac{\cos ^{2} \chi_{0}}{2} m_{\gamma^{\prime}}^{2} B_{\mu} B^{\mu}+j_{\mathrm{em}}^{\mu} A_{\mu},
\end{aligned}
$$

where $A_{\mu}, B_{\mu}$ are, respectively, the photon and the hidden photon fields, $F_{\mu \nu}$ and $B_{\mu \nu}$ their respective field strengths and $j_{\mathrm{em}}^{\mu}$ is the electromagnetic current. We have included

a hidden photon mass $m_{\gamma^{\prime}}$ which we will treat as a free parameter. Typical predicted values for the mixing angle $\chi_{0}$ in realistic string compactifications range between $10^{-16}$ and $10^{-2}[21]$ (see also $\left.[22,23]\right)$. 
The kinetic mixing term can be removed, leading to a canonical form of the kinetic lagrangian, by the following change of basis

$$
\{A, B\} \rightarrow\left\{A_{R}, S\right\}
$$

where

$$
\begin{aligned}
& A_{R}=\cos \chi_{0} A, \\
& S=B-\sin \chi_{0} A .
\end{aligned}
$$

Let us call $\gamma, \gamma_{s}$ the quanta of the $A_{R}$ and $S$ fields, respectively. The basis $\left\{\gamma, \gamma_{s}\right\}$ can be called the "interaction basis", since the photon $\gamma$ is the state that interacts with SM charged particles and $\gamma_{s}$, being orthogonal to $\gamma$, is completely sterile. In the interaction basis the kinetic lagrangian is diagonal but the mixing angle $\chi_{0}$ appears in an off-diagonal term in the mass-squared matrix,

$$
\mathcal{M}^{2}=\left(\begin{array}{cc}
m_{\gamma^{\prime}}^{2} \sin \chi_{0}^{2} & m_{\gamma^{\prime}}^{2} \sin \chi_{0} \cos \chi_{0} \\
m_{\gamma^{\prime}}^{2} \sin \chi_{0} \cos \chi_{0} & m_{\gamma^{\prime}}^{2} \cos ^{2} \chi_{0}
\end{array}\right) .
$$

The mass matrix $\mathcal{M}^{2}$ can be diagonalized through the unitary matrix $U$

$$
U=\left(\begin{array}{cc}
\cos \chi_{0} & -\sin \chi_{0} \\
\sin \chi_{0} & \cos \chi_{0}
\end{array}\right)
$$

that allows to identify the two "propagation states"

$$
\left(\begin{array}{l}
\gamma_{1} \\
\gamma_{2}
\end{array}\right)=U\left(\begin{array}{c}
\gamma \\
\gamma_{s}
\end{array}\right)
$$

where $\gamma_{1}$ is mostly photon-like and massless, while $\gamma_{2}$ has mass $m_{\gamma^{\prime}}$ and is close to the sterile state. The mismatch between the interaction $\left\{\gamma, \gamma_{s}\right\}$ and propagation $\left\{\gamma_{1}, \gamma_{2}\right\}$ states by the mixing angle $\chi_{0}$ is well-known to produce $\gamma \rightarrow \gamma_{s}$ oscillations [24], with a conversion probability in vacuum given by

$$
P_{\gamma \rightarrow \gamma_{s}}=\sin ^{2} 2 \chi_{0} \sin ^{2}\left(m_{\gamma^{\prime}}^{2} L / 4 \omega\right),
$$

where $L$ is the path length and $\omega$ is the photon energy.

In analogy to the neutrino case [25], the $\gamma \leftrightarrow \gamma_{s}$ oscillations are modified by the refraction properties of the medium. In the primordial plasma, photons acquire a nontrivial dispersion relation which can be parametrized by adding an effective photon mass $m_{\gamma}$ to the Lagrangian. This mass depends on the properties of the medium, as we will show explicitly in Section 4. This is generally complex, reflecting the absorption properties of the plasma, but in the context relevant for the present paper the imaginary part is negligible. In this case, the effective mixing angle is related to the vacuum by $[7,8]$

$$
\begin{aligned}
& \sin 2 \chi=\frac{\sin 2 \chi_{0}}{\sqrt{\sin ^{2} 2 \chi_{0}+\left(\cos 2 \chi_{0}-\xi\right)^{2}}}, \\
& \cos 2 \chi=\frac{\cos 2 \chi_{0}-\xi}{\sqrt{\sin ^{2} 2 \chi_{0}+\left(\cos 2 \chi_{0}-\xi\right)^{2}}},
\end{aligned}
$$

where the parameter

$$
\xi=m_{\gamma}^{2} / m_{\gamma^{\prime}}^{2}
$$

measures the significance of the medium effects. 


\section{Photon-Hidden Photon Oscillations in the Expanding Universe}

To obtain the bound on photon-hidden photon mixing, we have to compute the fraction of CMB photons that would oscillate into invisible $\gamma_{s}$. The photon effective mass squared $m_{\gamma}^{2}$ is generally proportional to the density of charged particles in the medium, so it relaxes as the photons propagate in the expanding primordial plasma. We are then facing a complicated problem of oscillations in an inhomogeneous medium.

For sufficiently early times, $m_{\gamma} \gg m_{\gamma^{\prime}}$ and, therefore, the photons are very close to be both interaction and propagation states at a given time. In this case, oscillations into $\gamma_{s}$ are suppressed,

$$
\xi \gg 1, \quad \chi \rightarrow \pi / 2 \quad \text { (medium suppression) . }
$$

As the universe expands, $\xi$ eventually reaches

$$
\xi=\cos \chi_{0}, \quad \chi \rightarrow \pi / 4 \quad \text { (resonance condition) . }
$$

When this condition is fulfilled, resonant photon-hidden photon conversions are possible, analogous to the well-known Mikheev-Smirnov-Wolfenstein (MSW) effect in the neutrino case [17-19]. As $\xi$ decreases below unity,

$$
\xi \ll 1, \quad \chi \rightarrow \chi_{0} \quad \text { (vacuum oscillations) }
$$

$\gamma \rightarrow \gamma_{s}$ oscillations take place as if they occurred in vacuum.

In vacuum, oscillations are proportional to $\sin ^{2} 2 \chi_{0}$ [see Eq. (8)] and since the blackbody nature of the CMB is established experimentally to an accuracy of $\simeq 10^{-4}$ we can easily exclude mixing angles $z 10^{-2}$. However, during a resonance $\sin 2 \chi \sim 1$ and the conversion $\gamma \rightarrow \gamma_{s}$ can be much stronger. In this paper we want to focus on the range of HP masses that can undergo such a resonant transition, and are, therefore, strongly constrained.

When the photon production and detection points are separated by many oscillation lengths, then on both sides of a resonance, $m_{\gamma}=m_{\gamma^{\prime}}$, the oscillation patterns wash out and the transition probability is given by [26]

$$
P_{\gamma \rightarrow \gamma_{s}}=\frac{1}{2}+\left(p-\frac{1}{2}\right) \cos 2 \chi_{0} \cos 2 \chi,
$$

where $\chi_{0}, \chi$ are the mixing angles at the detection and production points considered to be in vacuum and high density, respectively, and $p$ is the level crossing probability. This latter takes into account the deviation from adiabaticity of photon-HP oscillations in the resonance region. In particular, one has $p=0$ for a completely adiabatic transition and $p=1$ for an extremely nonadiabatic one.

As we will see, we are going to bound mixing angles much smaller than $\chi_{0} \sim 10^{-2}$, so for simplicity we can already take $\cos 2 \chi_{0} \simeq-\cos 2 \chi=1$ in Eq. (14) which, therefore, takes the much simpler form

$$
P_{\gamma \rightarrow \gamma_{s}} \simeq 1-p .
$$

The FIRAS sensitivity will allow us to bound $1-p \lesssim 10^{-4}$, requiring thus a non-adiabatic resonance. 
The crossing probability $p$ for photon-hidden photon resonant conversions can be obtained using the Landau-Zener expression

$$
p \simeq \exp \left(-2 \pi r k \sin ^{2} \chi_{0}\right),
$$

where $k=m_{\gamma^{\prime}}^{2} / 2 \omega$ is the $\gamma \rightarrow \gamma_{s}$ vacuum oscillation wavenumber and

$$
r=\left|\frac{d \ln m_{\gamma}^{2}(t)}{d t}\right|_{t=t_{\mathrm{res}}}^{-1}
$$

is a scale parameter to be evaluated at the location where a resonance occurs.

This expression has been widely used in solar and supernova neutrino oscillations [25]. It represents an accurate ansatz to calculate the level crossing probability for general density profiles (see, e.g. [27]). In particular, we observe that it reproduces the correct limits $p \simeq 0$ for $r \rightarrow \infty$ (adiabatic limit) and $p \simeq 1$ for $r \rightarrow 0$ (extreme non-adiabatic limit).

Finally, under the approximations outlined before, we can approximate the nonadiabatic conversion probability as

$$
P_{\gamma \rightarrow \gamma_{s}} \simeq 2 \pi r k \chi_{0}^{2}=\frac{\pi m_{\gamma^{\prime}}^{2} \chi_{0}^{2}}{\omega}\left|\frac{d \ln m_{\gamma}^{2}(t)}{d t}\right|_{t=t_{\mathrm{res}}}^{-1} .
$$

The half-width width of the resonance is, according to Eq. (9), $\delta \xi(t) \simeq \sin 2 \chi_{0}$, which corresponds to a time scale

$$
\tau_{r} \simeq r \sin 2 \chi_{0} .
$$

Due to the smallness of the vacuum mixing angle, the resonance is very narrow, so that it is reasonable to take into account the deviation from the adiabaticity only at the crossing point.

\section{Cosmological $m_{\gamma}$ profile}

From Eq. (18) it is clear that the only information needed to calculate the conversion probability is the profile of the photon effective mass $m_{\gamma}$ along the cosmological line of sight. The primordial plasma is thought to be electrically neutral and composed mainly of hydrogen and helium in a fraction per mass $Y_{p}=m_{\mathrm{He}} / m_{\mathrm{H}} \simeq 0.25$. The effective mass squared has a positive and a negative contribution [28] from scattering off free electrons and off neutral atoms $\ddagger$

$$
\begin{aligned}
m_{\gamma}^{2} & \simeq \omega_{\mathrm{P}}^{2}-2 \omega^{2}(n-1)_{\mathrm{H}} \\
& \simeq 1.4 \times 10^{-21}\left(X_{e}-7.3 \times 10^{-3}\left(\frac{\omega}{\mathrm{eV}}\right)^{2}\left(1-X_{e}\right)\right) \frac{n_{p}}{\mathrm{~cm}^{-3}} \mathrm{eV}^{2},
\end{aligned}
$$

where $\omega_{\mathrm{P}}^{2}=4 \pi \alpha n_{e} / m_{e}$ is the plasma frequency with $\alpha$ the fine structure constant, $m_{e}$ the electron mass and $n_{e}$ the free electron density. We have written the ionized fraction of hydrogen as $X_{e}=n_{e} / n_{p}$ with $n_{p}$ the proton density. The indices of refraction of neutral

$\ddagger$ The vacuum magnetic birefringence [29] due to a primordial magnetic field is negligible [30]. 
hydrogen and helium are $(n-1)_{\mathrm{H}}=13.6 \times 10^{-5}$ and $(n-1)_{\mathrm{He}}=3.48 \times 10^{-5}$ in normal conditions [28], rather insensitive to $\omega$. Since the fraction of electrons corresponding to helium is $\sim 13 \%$ and $(n-1)_{\mathrm{He}} \ll(n-1)_{\mathrm{H}}$ we have neglected the effects of helium in Eq. (20).

The photon frequency $\omega$ and the proton density $n_{p}$ are given in terms of redshift $z$, the photon energy today $\omega_{0}$, the CMB temperature today $T_{0}$ and the baryon to photon ratio $\eta \simeq 6.7 \times 10^{-10}$ as $[31]$

$$
\omega=\omega_{0}(1+z) ; n_{p}=\left(1-\frac{Y_{p}}{2}\right) \eta \frac{2 \zeta(3)}{\pi^{2}} T_{0}^{3}(1+z)^{3} .
$$

Note that for a negative effective mass squared a resonance is not possible. However, the negative contribution is proportional to $\omega^{2}\left(1-X_{e}\right)$ and thus is unimportant for sufficiently small frequencies (small $\omega_{0}$ or late times when the redshift $z$ is small) and/or large ionization fractions, $X_{e} \simeq 1$. Interestingly, the smallest frequencies are the most precisely determined by FIRAS.

Since the conversion probability Eq. (18) is proportional to $r$ and $\omega^{-1}$ which both grow with decreasing redshift, for a fixed HP mass the later the resonance the more adiabatic the transition will be and the stronger our constraints on the mixing parameter $\chi_{0}$ will be.

The history of the ionization fraction $X_{e}(z)$ is extremely complex. Above a temperature $T \sim 0.5 \mathrm{eV}$ (redshift $z \sim 1100$ ) hydrogen is fully ionized. As the universe temperature decreases, photons cannot ionize hydrogen efficiently and electrons and protons slowly combine. This makes the universe very transparent to radiation, indeed releasing the photon bath which we see today as the CMB. This epoch of so-called recombination has been studied in great detail in [32] from which we can take the values of $X_{e}$ as a function of redshift, shown in Fig. 1. Later on, the universe becomes ionized again due to ultraviolet radiation from the first quasars or population III stars. The 5 year data of the WMAP mission constrains the redshift of an instantaneous reionization to $z=11.0 \pm 1.4$ with $68 \%$ confidence level by CMB polarization studies $[1,2]$. At the same time reionization can be studied with the spectra of high redshift quasars, which show so-called Gunn-Peterson troughs due to absorption of light at Lyman- $\alpha$ frequencies. The study of several quasars by the Sloan Digital Sky Survey showed that reionization should end around a redshift $z \sim 6$, and, therefore, should be an extended process taking place between $z \sim 6-11$ [33].

In Fig. 1 we show a possible profile for the cosmological history of $X_{e}$ and $m_{\gamma}$ as a function of redshift z. We can learn several important things from this figure. Let us focus on the right panel. We can first compare the thin line, which corresponds to a cosmology when atoms are always ionized, i.e. $X_{e}=1$, with the more realistic colored lines which includes the ionization history of the left panel and assumes $\omega / T=1,3,4,10$, respectively. We see that during the dark ages $(6 \lesssim z \lesssim 1000), X_{e}$ drops very much and the resonances are moved to higher redshifts compared with the $X_{e}=1$ case. As we commented before, the later a resonance the stronger it is, and, therefore, the inclusion of the ionization history tends to decrease the bounds on $\chi_{0}$. 

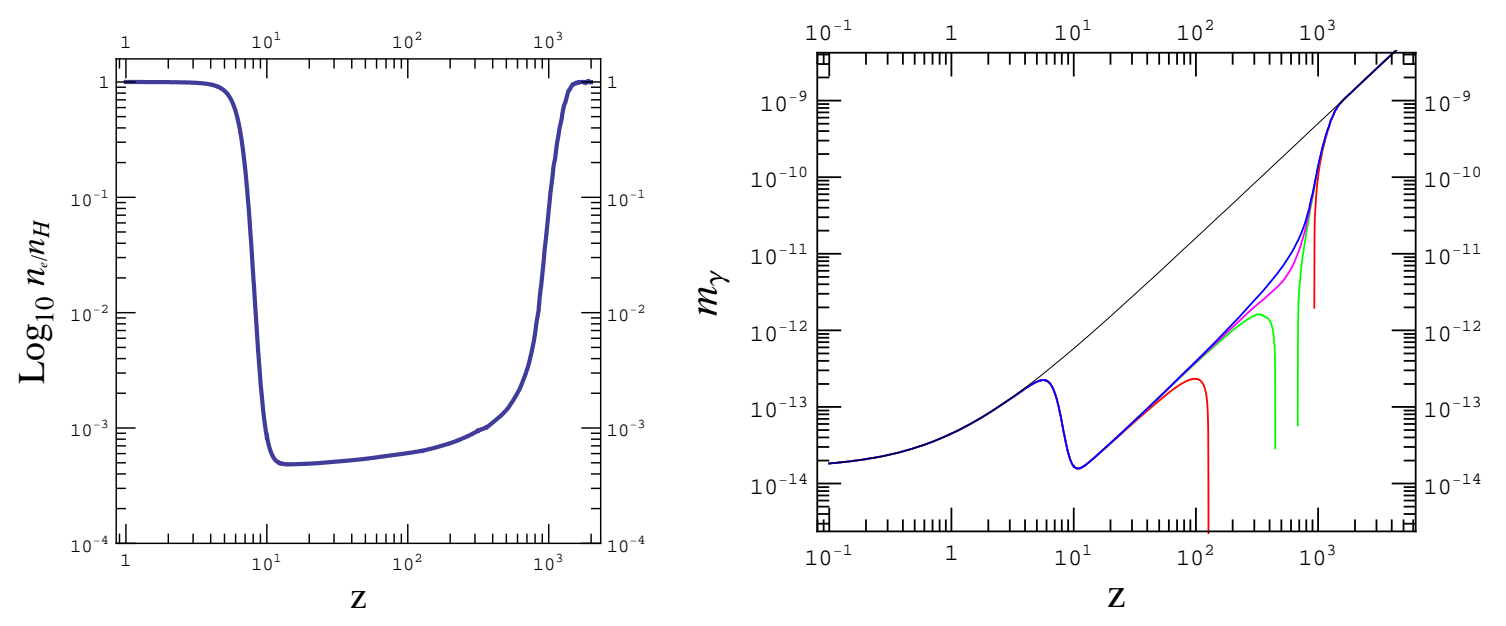

Figure 1. Left panel: The ionization fraction of hydrogen. For redshifts above $z=11$ the line is taken from the data of [32]. The re-ionization period between $z=6$ and $z=11$ is modeled. Right panel: Effective photon mass as function of redshift. The thin line uses $X_{e}=1$, the blue, magenta, green and red lines are for $\omega / T=1,3,4,10$, respectively. The two sharp dips bound the region where $m_{\gamma}$ becomes imaginary.

On the one hand, due to the smallness of $X_{e}$ during the dark ages, the index of refraction of neutral hydrogen can dominate over the contribution from free electrons turning the effective photon mass squared negative (see Eq. (20)). Since this negative contribution is proportional to the photon frequency squared, the region of redshifts for which this happens is broader for higher frequencies. From Fig. 1 we see that this does not happen for $\omega / T \lesssim 3$ but for $\omega / T=4$ it forbids a resonance in the range of redshifts $400 \lesssim z \lesssim 700$ and for $\omega / T=10$ the range without resonance is enlarged to $100 \lesssim z \lesssim 1000$.

Let us recall that this does not forbid a resonant transition but makes it happen at higher redshifts than those which correspond to $\omega / T \simeq 1$. Consider as an example the case $m_{\gamma^{\prime}}=4 \times 10^{-12} \mathrm{eV}$. For frequencies $\omega / T \lesssim 2$ the resonance, $m_{\gamma}=m_{\gamma^{\prime}}$, occurs at $z \sim 400$, for $\omega / T=4$ at $z \sim 700$ and for $\omega / T=10$ at $z \sim 1000$. Moreover, for these delayed resonances it becomes clear that the value of $r$, which is related to the inverse of the derivative of $m_{\gamma}$ is smaller than for resonances for low $\omega / T$. Because of these two facts, the resonances for high $\omega / T$ photons during the dark ages will be much less adiabatic than those for lower $\omega / T$ and, therefore, they will play a minor role in our bounds. There is only one possible exception to this conclusion. For very small masses $m_{\gamma^{\prime}} \lesssim 10^{-14} \mathrm{eV}$ there is no resonance at all for low $\omega / T$ but still there are resonances for $\omega / T \gtrsim 4$ during the dark ages. We have checked however that such resonances are extremely non-adiabatic and no interesting bounds can be derived from them. Therefore, in the following discussion we will not discuss resonances for $\omega / T \gtrsim 4$, although we will include them in our bounds.

Regarding the resonant conversion $\gamma \rightarrow \gamma_{s}$, and focusing on $\omega / T \lesssim 3$, we see three different possibilities in the homogeneous universe: 
- $m_{\gamma^{\prime}} \gtrsim 2 \times 10^{-13} \mathrm{eV}$ : there is only one resonance crossing

- $2 \times 10^{-13} \mathrm{eV} \lesssim m_{\gamma^{\prime}} \lesssim 10^{-14} \mathrm{eV}$ : there are three level crossings respectively after, during and before reionization.

- $m_{\gamma^{\prime}} \lesssim 10^{-14} \mathrm{eV}$ : no resonance is possible.

Our analytical ansatz for the crossing probability [Eq. (16)] has been shown to reproduce accurately the transitions also for nonmonotonic density profiles, where multiple crossings could arise (see, e.g., the supernova neutrino case [27]). In this case, assuming the factorization of $n$ different crossings, one obtains [27]

$$
p=\frac{1}{2}\left[1-\prod_{i=1, \cdots, n}\left(1-2 p_{i}\right)\right],
$$

and, therefore, assuming $1-p \lesssim 10^{-4}$ we can write

$$
P_{\gamma \rightarrow \gamma_{s}} \simeq 1-p \simeq \sum_{i} P_{\gamma \rightarrow \gamma_{s}}^{i}
$$

where the conversion probability for a resonance is still given by Eq. (18). As expected, since we know that photon depletion has to be very small, (and, therefore, also a possible re-creation) the dissappearence probabilities at different crossings just add up.

As commented previously, in the presence of multiple crossings $\left(2 \times 10^{-13} \mathrm{eV}\right.$ $\lesssim m_{\gamma^{\prime}} \lesssim 10^{-14} \mathrm{eV}$ ), the relevant crossing is the one occuring after reionization (at $z<6$ ), since the other two will be less adiabatic. This situation further simplifies our calculations. Since the crossings during reionization do not play a role, we need to evaluate them only for $z \lesssim 6$ (for $m_{\gamma^{\prime}}<2 \times 10^{-13} \mathrm{eV}$ ) and for, say, $z \gtrsim 70$ (for $\left.m_{\gamma^{\prime}}>2 \times 10^{-13} \mathrm{eV}\right)$.

During the epoch of matter domination, the small density inhomogeneities already present at $\mathrm{CMB}$ decoupling grow and the density profile along a line of sight can be quite complicated at the smallest redshifts, with lots of resonances at galactic length scales. However, according to Eq. (23) further resonances tend to increase the transition probability, thus tending to strengthen the constraints. It is, therefore, conservative to restrict oneself to the most adiabatic resonances in the smooth background, represented in Fig. 1.

Furthermore, the optical depth $\tau$ for crossing an object of typical size $l$ with number density $n$ along a cosmological line of sight of length $s \sim 4$ Gpc is $\tau \sim n l^{2} s \simeq$ $0.004\left(10^{-2} / \mathrm{Mpc}^{-3}\right)(l / 10 \mathrm{kpc})^{2}$ which is small for galactic objects. Within an order of magnitude this is consistent with galaxy number counts which yield $\sim 10^{8} \mathrm{sr}^{-1}$ [34]: Given that one galaxy subtends a solid angle $\Omega \sim\left(10 \mathrm{kpc} / 10^{3} \mathrm{Mpc}\right)^{2} \mathrm{sr} \sim 10^{-10} \mathrm{sr}$, this gives $\tau \sim 0.01$. Similarly, counts of galaxy cluster with mass $\gtrsim 10^{15} M_{\odot}$ yield $\sim 10^{-6} \mathrm{Mpc}^{-3}$ [35], or $\sim 10^{4} \mathrm{sr}^{-1}$. With a typical solid angle subtended of $\Omega \sim$ $\left(1 \mathrm{Mpc} / 10^{3} \mathrm{Mpc}\right)^{2} \mathrm{sr} \sim 10^{-6} \mathrm{sr}$, this also gives $\tau \sim 0.01$ for the average number of galaxy clusters crossed by a given line of sight. 


\subsection{Adiabaticity parameter}

Given our density profile it is now straightforward to compute the adiabaticity parameter $r$ for each crossing and from it, the photon disappearance probability. For masses $m_{\gamma^{\prime}}<10^{-14} \mathrm{eV}$ this would be very much dependent on the inhomogeneities at small redshifts, and we prefer not to treat this case. We are left with HP masses above the average effective cosmological plasma mass of photons at zero redshift.

Recall that in general $r \propto t$ so the most adiabatic crossing is the latest, when the universe expands slower and, therefore, sweeps smaller ranges of $m_{\gamma}$ in a given time. This argument also allows us to discard the effect of spatial inhomogeneities unless they have sizes comparable with the size of the universe. It also makes us neglect a possible back-reaction during the reionization crossing.

Let us then write,

$$
\frac{d \log m_{\gamma}^{2}}{d t}=\frac{d \log m_{\gamma}^{2}}{d z} \frac{d z}{d t}
$$

with

$$
\frac{d z}{d t}=-H_{0}(1+z) \sqrt{\Omega_{\lambda}+\Omega_{m}(1+z)^{3}+\Omega_{r}(1+z)^{4}},
$$

where we fix $H_{0}=70 \mathrm{~km} \mathrm{~s}^{-1} \mathrm{Mpc}^{-1}, \Omega_{m}=0.27, \Omega_{\lambda}=0.73, \Omega_{r}=6 \times 10^{-4}$ consistent with the recent determinations of cosmological parameters [33].

The function $d X_{e} / d z$ can be computed numerically. However, since the crossing during reionization does not play a role, we need this function only for $z \lesssim 6$ (for $m_{\gamma^{\prime}}<2 \times 10^{-13} \mathrm{eV}$ ) and for $z \gtrsim 70$ (for $m_{\gamma^{\prime}}>2 \times 10^{-13} \mathrm{eV}$ ). Below $z=6$ we can take it to zero, and for $z \gtrsim 70$ we can obtain it from the following fitting function

$$
\log _{10} X_{e}=-3.15 \frac{1}{e^{\frac{z-907}{160}}+1}
$$

valid at the \% level.

\section{FIRAS Bounds}

The CMB spectrum measured by FIRAS fits extremely well to a black body spectrum at a temperature $T_{0}=2.725 \pm 0.002[12]$. The energy range of the CMB spectrum measured by FIRAS [11] is $2.84 \times 10^{-4} \mathrm{eV} \leq \omega_{0} \leq 2.65 \times 10^{-3} \mathrm{eV}$, corresponding to $1.2 \lesssim \omega_{0} / T_{0} \lesssim 11.3$. In that region, the $\mathrm{CMB}$ blackbody becomes unprotected to distortions below a cosmic temperature $\sim \mathrm{keV}$, which corresponds to a photon mass of $\sim 10^{-4} \mathrm{eV}$. On the other hand, today the average plasma mass for photons is as low as $2 \times 10^{-14} \mathrm{eV}$. If HPs exist with a mass between these two values they will be produced resonantly and leave their imprint on the CMB.

The accuracy of FIRAS constraints $P_{\gamma \rightarrow \gamma_{s}} \lesssim f \simeq 10^{-4}$, which, using Eq. (18) leads to the bound

$$
\chi_{0} \lesssim\left(\frac{f \omega}{\pi r m_{\gamma^{\prime}}^{2}}\right)^{1 / 2} \simeq 1.1 \times 10^{-9} f^{1 / 2}\left(\frac{\mu \mathrm{eV}}{m_{\gamma^{\prime}}}\right)\left(\frac{\omega}{\mathrm{GHz}}\right)^{1 / 2}\left(\frac{\mathrm{pc}}{r}\right)^{1 / 2} .
$$




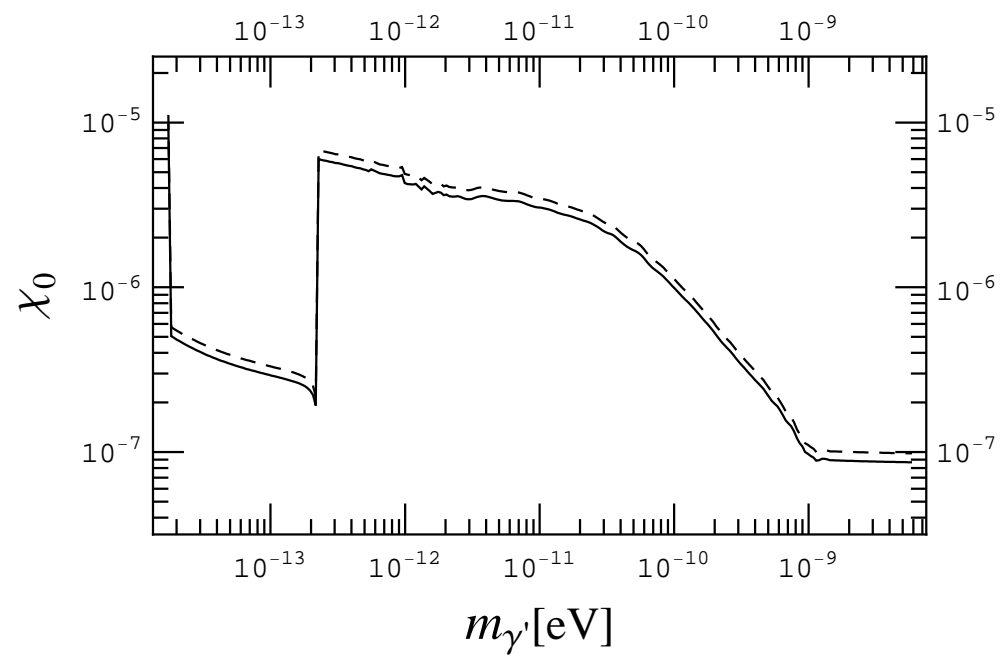

Figure 2. Bounds from distortions of the CMB blackbody due to $\gamma \rightarrow \gamma_{s}$ photon depletion: 95\% C.L. (solid) and 99\% C.L. (dashed).

In order to sharpen this bound, we have considered the distortion of the overall blackbody spectrum.

To this end we use the COBE-FIRAS data for the experimentally measured spectrum, corrected for foregrounds [11]. Note that the new calibration of FIRAS [12] is within the old errors and would not change any of our conclusions. The $N=43$ data points $\Phi_{i}^{\exp }$ at different frequencies $\omega_{i}$ are obtained by summing the best-fit blackbody spectrum to the residuals reported in Ref. [11]. The errors $\sigma_{i}^{\exp }$ are also available. In the presence of photon-hidden photon conversion, the original intensity of the "theoretical blackbody" at temperature $T$

$$
\Phi^{0}(\omega, T)=\frac{\omega^{3}}{2 \pi^{2}}[\exp (\omega / T)-1]^{-1}
$$

would be deformed to $\Phi\left(\omega, T, \chi_{0}, m_{\gamma^{\prime}}\right)=\Phi^{0}(\omega, T)\left[1-P_{\gamma \rightarrow \gamma_{s}}\left(\omega, \chi_{0}, m_{\gamma^{\prime}}\right)\right]$. We then build the reduced chi-squared function

$$
\chi_{\nu}^{2}(T, \lambda)=\frac{1}{N-1} \sum_{i}^{N}\left[\frac{\Phi_{i}^{\exp }-\Phi\left(\omega_{i}, T, \chi_{0}, m_{\gamma^{\prime}}\right)}{\sigma_{i}^{\exp }}\right]^{2} .
$$

We minimize this function with respect to $T$ for each point in the parameter space $\lambda=\left(m_{\gamma^{\prime}}, \chi_{0}\right)$, i.e. $T$ is an empirical parameter determined by the $\chi_{\nu}^{2}$ minimization for each $\lambda$ rather than being fixed at the standard value $T_{0}=2.725 \pm 0.002 \mathrm{~K}$.

In Fig. 2 we show our exclusion contour in the plane of $m_{\gamma^{\prime}}$ and $\chi_{0}$. The region above the continuous curve is the excluded region at $95 \%$ C.L., i.e. in this region the chance probability to experimentally obtain larger values of $\chi_{\nu}^{2}$ is lower than $5 \%$. We also show the corresponding $99 \%$ C.L. contour which is very close to the $95 \%$ contour so that another regression method and/or exclusion criterion would not change the results very much. Note that for masses $m_{\gamma^{\prime}}$ for which the resonant conversion takes place during the dark ages such a resonance is only possible for $\omega / T \lesssim 2$ for which the positive 
contribution to $m_{\gamma}^{2}$ dominates over the negative one. Only the lowest four points in the FIRAS data set satisfy $\omega / T<2$ and are thus distorted. On the other hand, for larger $\left(m_{\gamma^{\prime}}>10^{-10} \mathrm{eV}\right.$, cf. Fig. 1) or smaller masses $\left(2 \times 10^{-14} \mathrm{eV}<m_{\gamma^{\prime}}<2 \times 10^{-13} \mathrm{eV}\right)$, the resonance can happen up to $\omega / T=10$ and we can use the whole FIRAS data set. This explains why the bound we obtain is stronger in this latter region.

Finally, we comment that in our analysis we have assumed that hidden photons are produced only by oscillations. In this sense, we have neglected a primordial population of hidden photons, that could have subsequently been converted into photons, producing an additional distortion of the CMB. Our assumption is reasonable if we neglect the presence of primordial charged hidden particles, by whose annihilations hidden photons could have been produced.

\section{Astrophysical bounds}

The constraint of Eq. (27) holds for general astrophysical sources whose photon flux at frequency $\omega$ is known to deviate less than a fraction $f$ from a model prediction in the absence of mixing with hidden photons.

As long as the contribution of neutrals to $m_{\gamma}$ is negligible, the resonance condition is $m_{\gamma}^{2}=\omega_{\mathrm{P}}^{2}$. The resonance thus occurs at the plasma density

$$
n_{e} \simeq 7.3 \times 10^{8}\left(\frac{m_{\gamma^{\prime}}}{\mu \mathrm{eV}}\right)^{2} \mathrm{~cm}^{-3} .
$$

Such resonances can directly influence the photon flux only if the optical depth at the resonance is smaller than unity. Since the optical depth $\tau_{\gamma} \gtrsim \sigma_{\mathrm{T}} n_{e} r$ with $\sigma_{\mathrm{T}} \simeq$ $6.7 \times 10^{-25} \mathrm{~cm}^{2}$ the Thomson cross section, from Eq. (30) we obtain the condition

$$
r \lesssim 6.6 \times 10^{-4}\left(\frac{m_{\gamma^{\prime}}}{\mu \mathrm{eV}}\right)^{-2} \mathrm{pc}
$$

for the scale over which the mixing potential varies. Since furthermore only photons with frequency $\omega \gtrsim \omega_{\mathrm{P}}$ can propagate, we conclude from Eq. (27) that independent of the details of the astrophysical system, only mixing parameters satisfying

$$
\chi_{0} \gtrsim 5.3 \times 10^{-8} f^{1 / 2}\left(\frac{m_{\gamma^{\prime}}}{\mu \mathrm{eV}}\right)^{1 / 2}
$$

can be constrained by observing photon fluxes from astrophysical or cosmological objects. Note for example that for $m_{\gamma^{\prime}} \sim \mathrm{meV}$, the best possible bound from direct observations is thus $\chi_{0} \lesssim 1.7 \times 10^{-6} f^{1 / 2}$. Any mixing parameters smaller than this, therefore, has to be constrained by indirect methods (e.g. stellar cooling) or experimentally. This theoretical best possible bound from astrophysical sources is plotted for $f=1$ in Fig. 3. Our CMB based bounds are weaker than this best possible bound essentially because the scale $r$ in Eq. (27) over which the cosmological plasma mass varies is considerably smaller than the photon mean free path Eq. (31) that would lead to the most adiabatic resonance possible for unabsorbed photons. 


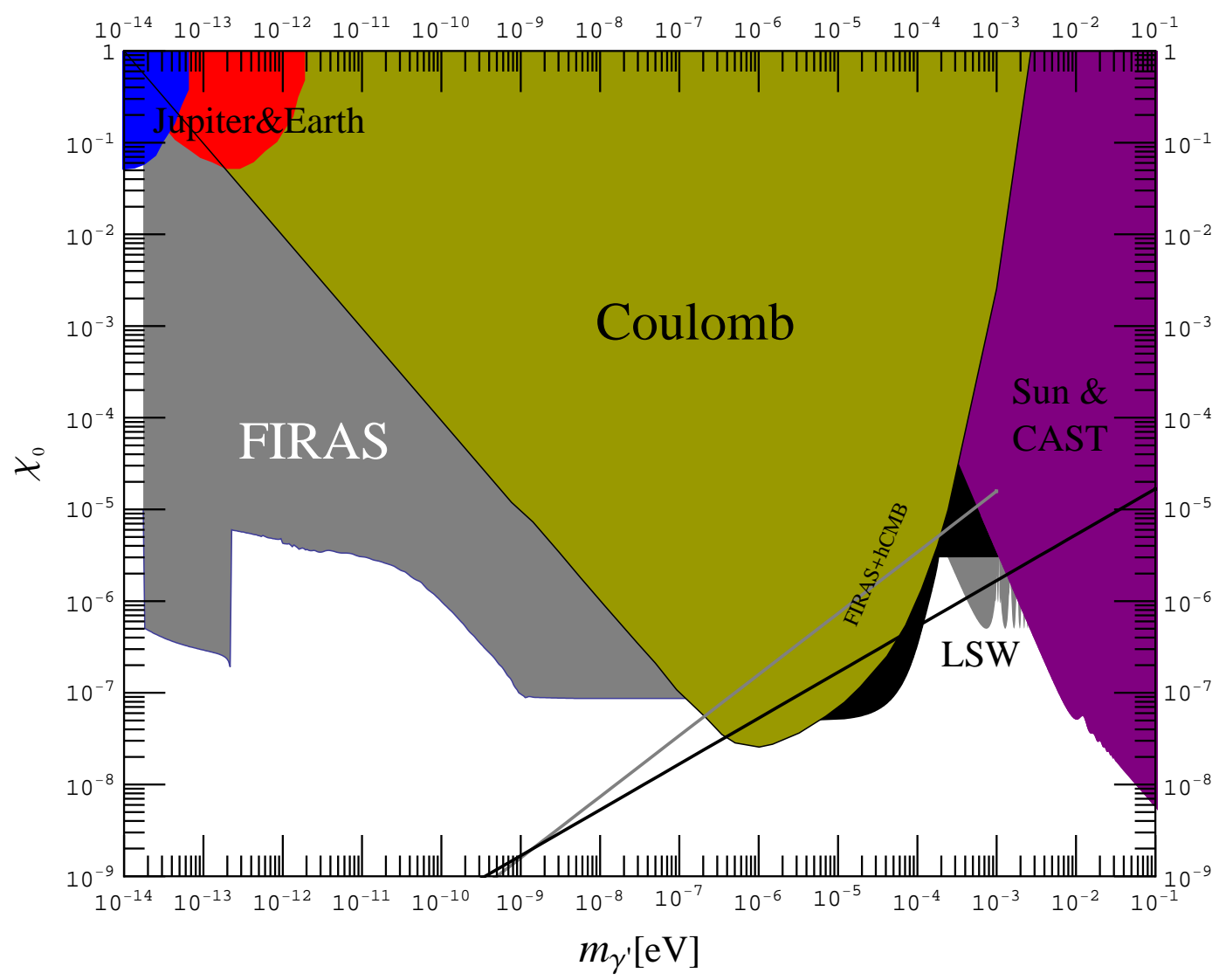

Figure 3. Bounds from resonant $\gamma \rightarrow \gamma_{s}$ depletion of the CMB blackbody as constrained by FIRAS data in this work (gray region) and in [8] (black region). The gray diagonal line separates the region where the resonance happens at small damping (left, this work) or at strong damping (right, cf. [8]). Plotted for comparison are bounds from tests of the Coulomb $1 / r^{2}$ law [36,37], magnetic fields of Jupiter and earth [38], photon-regeneration-experiments [39-44], arguments of the lifetime of the Sun and the CAST search of solar axions $[7,45]$. The solid black line indicates the best possible bound Eq. (32) that can be obtained from astrophysical or cosmological sources whose photon flux is known to be unmodified by photon-HP mixing to order unity.

\section{Conclusions}

In this paper, we have revisited the bounds on photon-hidden photon oscillations coming from cosmology, deriving updated constraints from the high precision CMB spectrum data collected by the FIRAS instrument on board of COBE. We have also commented about complementary bounds from a depletion of the photon flux from astrophysical sources. Previous studies $[3,13]$ were derived in the pre-COBE era and/or more importantly they lacked a detailed treatment of the effects of the plasma medium on the photon-hidden photon oscillations. This has motivated us to re-evaluate the bounds. This problem has presented interesting analogies to similar situations encountered in neutrino oscillation physics. In this regard, we had the benefit to apply to our case different analytical recipes developed in neutrino oscillation studies. 
The result of our analysis leads to a bound on the mixing angle $\chi_{0} \lesssim 10^{-7}-10^{-5}$ for hidden photon masses between $10^{-14} \mathrm{eV}$ and $10^{-7} \mathrm{eV}$. In Fig. 3 we plot our FIRAS bound together with the other ones existing in the literature (see [46] for a complete review). It turns out that our new cosmological bound excludes a region of low-mass and low-mixing angle in the hidden photon parameter space, that was unconstrained by previous arguments. As a result of our new bound, it is unlikely that hidden photons with masses smaller than $10^{-7} \mathrm{eV}$ can play a cosmological role. Conversely, for meV masses, resonant photon-hidden photon oscillations happen after nucleosynthesis but before CMB decoupling, increasing the effective number of neutrinos but also the baryon to photon ratio with interesting cosmological consequences [8]. The mixing angles required for this effect could be probed in current laboratory experiments.

We comment that our cosmological bound on hidden photons is based on an approximate treatment of the plasma environment. This would leave open possible improvements of our limit when a more accurate description of the primordial plasma will be achieved. Moreover, further improvements of our cosmological bound for

extremely small masses and mixing could be reached observing a possible depletion of photons from astrophysical sources, as we proposed in Sec. 6. In this context, for example it still remains to investigate if photon-hidden photon oscillations could have some impact on the apparent dimming of supernovae Ia, mimicking the acceleration of the universe.

\section{Acknowledgements}

This work was supported by the Deutsche Forschungsgemeinschaft (SFB 676 "Particles, Strings and the Early Universe: The Structure of Matter and Space-Time) and by the European Union (contracts No. RII3-CT-2004-506222). The work of A.M. is supported by the Italian Istituto Nazionale di Fisica Nucleare (INFN). J.R. wishes to thank A. Ringwald for conversations and the Max Planck Institute for Physics in Munich for hospitality during the realization of this work. We also thank Georg Raffelt for careful reading the manuscript and for useful remarks on it. 


\section{References}

[1] WMAP Collaboration, G. Hinshaw et al., "Five-Year Wilkinson Microwave Anisotropy Probe (WMAP 1) Observations: Data Processing, Sky Maps, \& Basic Results," arXiv:0803.0732 [astro-ph].

[2] WMAP Collaboration, M. R. Nolta et al., "Five-Year Wilkinson Microwave Anisotropy Probe (WMAP) Observations: Angular Power Spectra," arXiv:0803.0593 [astro-ph].

[3] H. Georgi, P. H. Ginsparg, and S. L. Glashow, "Photon oscillations and the cosmic background radiation," Nature 306 (1983) 765-766.

[4] J. Jaeckel and A. Ringwald, "A Cavity Experiment to Search for Hidden Sector Photons," Phys. Lett. B659 (2008) 509-514, arXiv:0707.2063 [hep-ph].

[5] S. N. Gninenko and J. Redondo, "On search for eV hidden sector photons in Super-Kamiokande and CAST experiments," Phys. Lett. B664 (2008) 180-184, arXiv:0804.3736 [hep-ex].

[6] J. Jaeckel and J. Redondo, "Searching Hidden-sector Photons inside a Superconducting Box," Europhys. Lett. 84 (2008) 31002, arXiv:0806.1115 [hep-ph].

[7] J. Redondo, "Helioscope Bounds on Hidden Sector Photons," JCAP 0807 (2008) 008, arXiv:0801.1527 [hep-ph].

[8] J. Jaeckel, J. Redondo, and A. Ringwald, "Signatures of a hidden cosmic microwave background," Phys. Rev. Lett. 101 (2008) 131801, arXiv:0804.4157 [astro-ph].

[9] H.-S. Zechlin, D. Horns, and J. Redondo, "New Constraints on Hidden Photons using Very High Energy Gamma-Rays from the Crab Nebula," arXiv:0810.5501 [astro-ph].

[10] M. Postma and J. Redondo, "Massive hidden photons as lukewarm dark matter," arXiv:0811.0326 [hep-ph].

[11] D. J. Fixsen et al., "The Cosmic Microwave Background Spectrum from the Full COBE/FIRAS Data Set," Astrophys. J. 473 (1996) 576, arXiv:astro-ph/9605054.

[12] J. C. Mather, D. J. Fixsen, R. A. Shafer, C. Mosier, and D. T. Wilkinson, "Calibrator Design for the COBE Far Infrared Absolute Spectrophotometer (FIRAS)," Astrophys. J. 512 (1999) 511-520, arXiv:astro-ph/9810373.

[13] H. P. Nordberg and G. F. Smoot, "The Cosmic Microwave Background Spectrum:an Analysis of Observations," arXiv:astro-ph/9805123.

[14] A. Mirizzi, G. G. Raffelt, and P. D. Serpico, "Photon axion conversion as a mechanism for supernova dimming: Limits from CMB spectral distortion," Phys. Rev. D72 (2005) 023501, arXiv: astro-ph/0506078.

[15] A. Mirizzi, D. Montanino, and P. D. Serpico, "Revisiting cosmological bounds on radiative neutrino lifetime," Phys. Rev. D76 (2007) 053007, arXiv:0705.4667 [hep-ph].

[16] A. Melchiorri, A. Polosa, and A. Strumia, "New bounds on millicharged particles from cosmology," Phys. Lett. B650 (2007) 416-420, arXiv:hep-ph/0703144.

[17] L. Wolfenstein, "Neutrino oscillations in matter," Phys. Rev. D17 (1978) 2369-2374.

[18] S. P. Mikheyev and A. Y. Smirnov, "Resonance enhancement of oscillations in matter and solar neutrino spectroscopy," Yad. Fiz. (1985) no. 42, 1441. [Sov. J. Nucl. Phys. 42, 913 (1985)].

[19] S. P. Mikheyev and A. Y. Smirnov, "Resonant amplification of neutrino oscillations in matter and solar neutrino spectroscopy," Nuovo Cimento $C$ (1986) no. 9, 17.

[20] B. Holdom, "Two U(1) 's AND epsilon charge shifts," Phys. Lett. B166 (1986) 196.

[21] K. R. Dienes, C. F. Kolda, and J. March-Russell, "Kinetic mixing and the supersymmetric gauge hierarchy," Nucl. Phys. B492 (1997) 104-118, hep-ph/9610479.

[22] S. A. Abel, J. Jaeckel, V. V. Khoze, and A. Ringwald, "Illuminating the hidden sector of string theory by shining light through a magnetic field," Phys. Lett. B666 (2008) 66-70, arXiv: hep-ph/0608248.

[23] S. A. Abel, M. D. Goodsell, J. Jaeckel, V. V. Khoze, and A. Ringwald, "Kinetic Mixing of the Photon with Hidden U(1)s in String Phenomenology," JHEP 07 (2008) 124, arXiv:0803.1449 [hep-ph]. 
[24] L. B. Okun, "Limits of electrodynamics: paraphotons?," Sov. Phys. JETP 56 (1982) 502.

[25] T.-K. Kuo and J. T. Pantaleone, "Neutrino Oscillations in Matter," Rev. Mod. Phys. 61 (1989) 937.

[26] S. J. Parke, "Nonadiabatic Level Crossing in Resonant Neutrino Oscillations," Phys. Rev. Lett. 57 (1986) 1275-1278.

[27] G. L. Fogli, E. Lisi, D. Montanino, and A. Mirizzi, "Analysis of energy- and time-dependence of supernova shock effects on neutrino crossing probabilities," Phys. Rev. D68 (2003) 033005, arXiv: hep-ph/0304056.

[28] M. Born and E. Wolf, Principles of Optics. Pergamon Press, 1980. 691-692.

[29] S. L. Adler, "Photon splitting and photon dispersion in a strong magnetic field," Annals Phys. 67 (1971) 599-647.

[30] T. Yanagida and M. Yoshimura, "Resonant axion - photon conversion in the early universe," Phys. Lett. B202 (1988) 301.

[31] E. W. Kolb and M. S. Turner, The Early universe, vol. 69 of Front. Phys. 1990.

[32] S. Seager, D. D. Sasselov, and D. Scott, "A New Calculation of the Recombination Epoch," arXiv: astro-ph/9909275.

[33] WMAP Collaboration, J. Dunkley et al., "Five-Year Wilkinson Microwave Anisotropy Probe (WMAP) Observations: Likelihoods and Parameters from the WMAP data," arXiv:0803.0586 [astro-ph].

[34] R. F. N. Metcalfe, T. Shanks and N. Roche, "Galaxy number counts - III. Deep CCD observations to B=27.5 mag," Mon. Not. Roy. Astron. Soc. (1995) no. 273, 257.

[35] A. Jenkins et al., "Mass function of dark matter halos," Mon. Not. Roy. Astron. Soc. (2001) no. 321,372 .

[36] D. F. Bartlett and S. Loegl, "Limits on an electromagnetic fifth force," Phys. Rev. Lett. 61 (1988) 2285-2287.

[37] E. R. Williams, J. E. Faller, and H. A. Hill, "New experimental test of Coulomb's law: A Laboratory upper limit on the photon rest mass," Phys. Rev. Lett. 26 (1971) 721-724.

[38] A. S. Goldhaber and M. M. Nieto, "Terrestrial and extra-terrestrial limits on the photon mass," Rev. Mod. Phys. 43 (1971) 277-296.

[39] LIPSS Collaboration, A. Afanasev et al., "New Experimental Limit on Photon Hidden-Sector Paraphoton Mixing," arXiv:0810.4189 [hep-ex].

[40] M. Ahlers, H. Gies, J. Jaeckel, J. Redondo, and A. Ringwald, "Laser experiments explore the hidden sector," Phys. Rev. D77 (2008) 095001, arXiv:0711.4991 [hep-ph].

[41] M. Ahlers, H. Gies, J. Jaeckel, J. Redondo, and A. Ringwald, "Light from the Hidden Sector," Phys. Rev. D76 (2007) 115005, arXiv:0706.2836 [hep-ph].

[42] BRFT Collaboration, R. Cameron et al., "Search for nearly massless, weakly coupled particles by optical techniques," Phys. Rev. D47 (1993) 3707-3725.

[43] GammeV (T-969) Collaboration, A. S. Chou et al., "Search for axion-like particles using a variable baseline photon regeneration technique," Phys. Rev. Lett. 100 (2008) 080402, arXiv:0710.3783 [hep-ex].

[44] BMV Collaboration, M. Fouche et al., "Search for photon oscillations into massive particles," Phys. Rev. D78 (2008) 032013, arXiv:0808.2800 [hep-ex].

[45] CAST Collaboration, S. Andriamonje et al., "An improved limit on the axion-photon coupling from the CAST experiment," JCAP 0704 (2007) 010, hep-ex/0702006.

[46] J. Redondo, "The low energy frontier: probes with photons," arXiv:0805.3112 [hep-ph]. 